\title{
Group Classification of the General Evolution Equation: Local and Quasilocal Symmetries
}

\author{
Renat ZHDANOV ${ }^{\dagger}$ and Victor LAHNO ${ }^{\ddagger}$ \\ † Institute of Mathematics, 3 Tereshchenkivs'ka Str., Kyiv 4, 01601 Ukraine \\ E-mail: renat@imath.kiev.ua \\ ¥ State Pedagogical University, 2 Ostrogradskogo Str., Poltava, 36003 Ukraine \\ E-mail: laggo@poltava.bank.gov.ua
}

Received September 04, 2005, in final form October 19, 2005; Published online October 25, 2005

Original article is available at http://www.emis.de/journals/SIGMA/2005/Paper009/

\begin{abstract}
We give a review of our recent results on group classification of the most general nonlinear evolution equation in one spatial variable. The method applied relies heavily on the results of our paper Acta Appl. Math., 69, 2001, in which we obtain the complete solution of group classification problem for general quasilinear evolution equation.
\end{abstract}

Key words: group classification; symmetry; second order nonlinear evolution equation 2000 Mathematics Subject Classification: 35K55; 58J70

In this paper we briefly review our recent results on group classification of the general nonlinear evolution equation

$$
u_{t}=F\left(t, x, u, u_{x}, u_{x x}\right)
$$

Here $u=u(t, x), u_{t}=\partial u / \partial t, u_{x}=\partial u / \partial x, u_{x x}=\partial^{2} u / \partial x^{2} ; F$ is an arbitrary smooth function obeying the restriction $\partial F / \partial u_{x x} \neq 0$.

Using the standard Lie approach (see, e.g., [1, 2, 3, 4]) we prove that the maximal invariance group of equation (1) is generated by the operator

$$
\mathrm{Q}=\tau(t) \partial_{t}+\xi(t, x, u) \partial_{x}+\eta(t, x, u) \partial_{u}
$$

where functions $\tau, \xi$ and $\eta$ are arbitrary solutions of a single partial differential equation (PDE)

$$
\begin{aligned}
\eta_{t}- & u_{x} \xi_{t}+\left(\eta_{u}-\tau_{t}-u_{x} \xi_{u}\right) F=\left(\eta_{x}+u_{x}\left(\eta_{u}-\xi_{x}\right)-u_{x}^{2} \xi_{u}\right) F_{u_{x}} \\
& +\left(\eta_{x x}+u_{x}\left(2 \eta_{x u}-\xi_{x x}\right)+u_{x}^{2}\left(\eta_{u u}-2 \xi_{x u}\right)-u_{x}^{3} \xi_{u u}\right. \\
& \left.+u_{x x}\left(\eta_{u}-2 \xi_{x}\right)-3 u_{x} u_{x x} \xi_{u}\right) F_{u_{x x}}+\tau F_{t}+\xi F_{x}+\eta F_{u} .
\end{aligned}
$$

So to obtain (exhaustive) group classification of the class of equations (1) we need to construct all possible functions, $\tau, \xi, \eta$ and $F$, obeying the above constraint (determining equation). Evidently the challenge of the problem is in the word all, since the system of classifying equations is not over-determined (as is customary for this type of problems). Moreover, it is underdetermined. This is the reason why the numerous papers devoted to group classification of nonlinear evolution equations $[5,6,7,8,9,10,11,12,13,14]$ deal mostly with classes of PDEs depending on arbitrary functions of one, or at most two, variables.

A starting point of our analysis is a simple observation that solutions $\boldsymbol{v}_{a}=\left(\tau_{a}, \xi_{a}, \eta_{a}\right)$, $a=1, \ldots, n$, of $(3)$ span a Lie algebra $\ell$. So without any loss of generality we can replace (3) 
with the finite set of systems of PDEs

Equation (3),

$$
\left[Q_{i}, Q_{j}\right]=C_{i j}^{k} Q_{k}
$$

or, equivalently,

$$
\begin{aligned}
& \text { Equation (3), } \\
& \begin{array}{l}
Q_{i} \tau_{j}-Q_{j} \tau_{i}=C_{i j}^{k} \tau_{k}, \\
Q_{i} \xi_{j}-Q_{j} \xi_{i}=C_{i j}^{k} \xi_{k}, \\
Q_{i} \eta_{j}-Q_{j} \eta_{i}=C_{i j}^{k} \eta_{k} .
\end{array}
\end{aligned}
$$

In the above formulas the indices $i, j, k$ take the values $1, \ldots, n$ ( $n \geq 1$ is a dimension of the corresponding Lie algebra), and $C_{i j}^{k}$ are structure constants of the Lie algebra $\ell$.

If we solve the (over-determined) system of PDEs (4) for all possible dimensions $n \geq 1$ of all admissible Lie algebras, $\ell$, then the problem of group classification of equation (1) is completely solved. In other words, the problem of group classification of the general evolution equation (1) reduces to integrating over-determined systems of PDEs (4) for all $n=1,2, \ldots, n_{0}$, where $n_{0}$ is the maximal dimension of the Lie algebra admitted by the equation under study.

One way to handle the above problem would be starting with investigating compatibility of systems (4) for all $n \geq 1$. This strategy is close in spirit to Reid's procedure of describing the algebra admitted by PDE without integrating determining equations [16].

However, a more natural approach is actually to integrate equations (4) so that compatibility conditions come as a by-product. This is even more so if we take into account that lowdimensional abstract Lie algebras are described up to the dimension $n=6$ (mainly due to efforts by Mubarakzyanov [17, 18]). So, if we

1. construct all realizations of Lie algebras by operators, the coefficients of which satisfy equation (3), up to some fixed dimension $n_{0}$, and

2. prove that (1) does not admit invariance algebras of the dimension $n>n_{0}$,

then the problem of group classification of equation (1) is completely solved.

The underlying ideas of the above approach are rather natural. No wonder that they have already been used in various contexts. In particular Fushchych \& Serov [19] exploited them to classify conformally-invariant wave equations in the multidimensional case. In a more systematic way these ideas have been utilized by Gagnon \& Winternitz [20] to classify variable coefficient Schrödinger equations.

In its present form the approach formulated above has been developed in [21], where we perform preliminary group classification of nonlinear Schrödinger equations. Later we applied this approach to classify second-order quasilinear evolution equations [22, 23], third-order evolution equations [24] and nonlinear wave equations [25] in one spatial variable.

We perform group classification within the action of equivalence group preserving the class of PDEs under study. It is not difficult to prove that the maximal equivalence transformation group preserving class (1) is

$$
\bar{t}=T(t), \quad \bar{x}=X(t, x, u), \quad \bar{u}=U(t, x, u),
$$

where

$$
T^{\prime}=\frac{d T}{d t} \neq 0, \quad \frac{D(X, U)}{D(x, u)} \neq 0 .
$$


In the paper [22] we obtain an exhaustive group classification of (quasilinear) evolution equation

$$
v_{t}=f\left(t, x, v, v_{x}\right) v_{x x}+g\left(t, x, v, v_{x}\right), \quad v=v(t, x) .
$$

Those results provide almost complete solution of the problem of group classification for general evolution equation (1). By this we mean that if equation (1) admits a one-parameter transformation group with the infinitesimal generator

$$
Q=\xi(t, x, u) \partial_{x}+\eta(t, x, u) \partial_{u},
$$

then it is transformed into an equation of the form (6). Consequently, if the symmetry algebra of (1) contains at least one operator of the form (7) then it is equivalent to the PDE (6). What is more, the symmetry algebra of (1) is mapped into the symmetry algebra of (6). Note, however, that some symmetry operators may become nonlocal (see the examples below).

Indeed operator (7) can be reduced to the canonical form $\partial_{u^{\prime}}$ by a suitable change of variables

$$
t^{\prime}=T(t), \quad x^{\prime}=X(t, x, u), \quad u^{\prime}=U(t, x, u)
$$

(note that the above transformation belongs to the equivalence group of equation (1)). The corresponding invariant equation takes the form

$$
u_{t^{\prime}}^{\prime}=F^{\prime}\left(t^{\prime}, x^{\prime}, u_{x^{\prime}}^{\prime}, u_{x^{\prime} x^{\prime}}^{\prime}\right) .
$$

Differentiating the obtained equation by $x^{\prime}$, replacing $u_{x^{\prime}}^{\prime}$ with $v\left(t^{\prime}, x^{\prime}\right)$ and dropping the primes we arrive at the quasilinear PDE of the form (6).

Before proceeding to exploit the above fact any further, we briefly summarize the principal results of [22].

- There are 2 inequivalent PDEs (6) admitting one-dimensional algebras.

- There are 5 inequivalent PDEs (6) admitting two-dimensional algebras.

- There are 34 inequivalent PDEs (6) admitting three-dimensional algebras.

- There are 35 inequivalent PDEs (6) admitting four-dimensional algebras.

- There are 6 inequivalent PDEs (6) admitting five-dimensional algebras.

Note that there are PDEs (1) admitting infinite-dimensional symmetry algebras. However these equations are locally equivalent to linear PDEs and therefore their infinite-dimensional symmetries provide no essential information about them. That is why these PDEs do not appear in the above list. Note also that group classification of the general linear parabolic type equation have been performed by Sophus Lie [26] (these results can also be found in [1]).

As an example, we give below the complete list of inequivalent equations (6) invariant under five-dimensional Lie algebras:

$$
\begin{aligned}
& u_{t}=u^{-4} u_{x x}-2 u^{-5} u_{x}^{2}, \\
& u_{t}=u_{x x}+x^{-1} u u_{x}-x^{-2} u^{2}-2 x^{-2} u, \\
& u_{t}=u_{x}^{-2} u_{x x}+u_{x}^{-1} \\
& u_{t}=\mathrm{e}^{u_{x}} u_{x x}, \\
& u_{t}=u_{x}^{n} u_{x x}, \quad n \geq-1, \quad n \neq 0, \\
& u_{t}=\left(1+u_{x}^{2}\right)^{-1} \exp \left(n \arctan u_{x}\right) u_{x x} .
\end{aligned}
$$


It follows from the above considerations that, if the invariance algebra of equation (1) contains an operator of the form (7), then it is equivalent to equation (6) the group properties of which are already known. Thus to complete group classification of equation (1) we need to describe all equations (1) the invariance algebras of which are spanned by the operators

$$
Q_{i}=\tau_{i}(t) \partial_{t}+\xi_{i}(t, x, u) \partial_{x}+\eta_{i}(t, x, u) \partial_{u}, \quad i=1, \ldots, n,
$$

where the functions $\tau_{1}(t), \ldots, \tau_{n}(t)$ are linearly independent. We denote the class of such equations as $\mathcal{L}_{1}$.

We prove that the highest dimension of an invariance algebra of equation (6) belonging to $\mathcal{L}_{1}$ equals 3 . The algebra in question is $s l(2, \mathbb{R})$ and the corresponding invariant equations are given below:

$$
\begin{gathered}
u_{t}=x^{-1} u u_{x}-x^{-2} u^{2}+x^{-2} \tilde{F}\left(x^{2} u_{x x}-2 u, 2 u-x u_{x}\right), \\
s l(2, \mathbb{R})=\left\langle 2 t \partial_{t}+x \partial_{x},-t^{2} \partial_{t}-t x \partial_{x}+x^{2} \partial_{u}, \partial_{t}\right\rangle \\
u_{t}=-\frac{1}{4} x^{-1} u_{x}+x^{-3} u_{x}^{-1} \tilde{F}\left(u, u_{x}^{-2} u_{x x}+3 x^{-1} u_{x}^{-1}\right) \\
s l(2, \mathbb{R})=\left\langle 2 t \partial_{t}+x \partial_{x},-t^{2} \partial_{t}+x\left(x^{2}-t\right) \partial_{x}, \partial_{t}\right\rangle .
\end{gathered}
$$

Here $\tilde{F}$ is an arbitrary smooth function.

There are only two equations from $\mathcal{L}_{1}$ admitting lower-dimensional invariance algebras, namely,

$$
\begin{aligned}
& u_{t}=\tilde{F}\left(x, u^{-1} u_{x}, u^{-1} u_{x x}\right), \quad \ell=\left\langle-t \partial_{t}-u \partial_{u}, \partial_{t}\right\rangle . \\
& u_{t}=\tilde{F}\left(x, u, u_{x}, u_{x x}\right), \quad \ell=\left\langle\partial_{t}\right\rangle .
\end{aligned}
$$

In the above formulas $\tilde{F}$ is an arbitrary smooth function.

Equations from $\mathcal{L}_{1}$ together with invariant equations of the form (6) provide the complete solution of the problem of classifying equations (1) that admit nontrivial Lie symmetry.

As we noted in [23], results of the group classification of (1) can be utilized to derive their quasilocal symmetries. The term quasilocal has been introduced independently in [27] and [28] to distinguish nonlocal symmetries that are equivalent to local ones through nonlocal transformations.

We have already shown that equations (1) and (6) are related through the nonpoint transformation $v(t, x)=u_{x}(t, x)$ or, inversely, $u(t, x)=\partial_{x}^{-1} v(t, x)$. Suppose now that equation (1) admits the one-parameter transformation group

$$
\begin{aligned}
& t^{\prime}=T(t, \theta), \\
& x^{\prime}=X(t, x, u, \theta), \\
& u^{\prime}=U(t, x, u, \theta) .
\end{aligned}
$$

Computing the first prolongation of the above formulas gives the transformation rule for the first derivative of $u^{\prime}$

$$
\frac{\partial u^{\prime}}{\partial x^{\prime}}=\frac{u_{x} U_{u}+U_{x}}{u_{x} X_{u}+X_{x}} .
$$

In terms of the variables, $t, x, v(t, x)$, this transformation group is

$$
\begin{aligned}
t^{\prime} & =T(t, \theta), \\
x^{\prime} & =X(t, x, u, \theta), \\
v^{\prime} & =\frac{v U_{u}(t, x, u(t, x), \theta)+U_{x}(t, x, u(t, x), \theta)}{v X_{u}(t, x, u(t, x), \theta)+X_{x}(t, x, u(t, x), \theta)},
\end{aligned}
$$


where $u(t, x)=\partial^{-1} v(t, x)$. Consequently, if the relation

$$
X_{u u}^{2}+X_{x u}^{2}+U_{u u}^{2}+U_{u x}^{2} \neq 0
$$

holds, the transformed equation (6) possesses a quasilocal symmetry. If a symmetry group of equation (1) satisfies constraint (10), then we say that this equation belongs to the class $\mathcal{L}_{2}$. In what follows we describe all equations from $\mathcal{L}_{2}$, the symmetry algebras of which are at most three-dimensional.

It is not difficult to become convinced of the fact that the class $\mathcal{L}_{2}$ does not contain equations the maximal invariance algebras of which are of the dimension $n \leq 2$. Below we give a full list of inequivalent equations belonging to $\mathcal{L}_{2}$ and admitting three-dimensional Lie algebras (we follow notations of [23]).

Algebra $s l(2, \mathbb{R})$ :

1. Realization

$$
Q_{1}=\partial_{u}, \quad Q_{2}=2 u \partial_{u}-x \partial_{x}, \quad Q_{3}=-u^{2} \partial_{u}+x u \partial_{x}
$$

Invariant equation:

$$
u_{t}=x u_{x} \tilde{F}\left(t, x^{-5} u_{x}^{-3} u_{x x}+2 x^{-6} u_{x}^{-2}\right) .
$$

2. Realization

$$
Q_{1}=\partial_{u}, \quad Q_{2}=2 u \partial_{u}-x \partial_{x}, \quad Q_{3}=\left(\varepsilon x^{-4}-u^{2}\right) \partial_{u}+x u \partial_{x}, \quad \varepsilon= \pm 1 .
$$

Invariant equation:

$$
u_{t}=x^{-2} \sqrt{x^{6} u_{x}^{2}+4 \varepsilon} \tilde{F}\left(t,\left(x^{6} u_{x}^{2}+4 \varepsilon\right)^{-\frac{3}{2}}\left(x^{4} u_{x x}+5 x^{3} u_{x}+\frac{1}{2} x^{9} u_{x}^{3}\right)\right) .
$$

\section{Algebra so(3):}

1. Realization

$Q_{1}=\partial_{u}, \quad Q_{2}=\cos u \partial_{x}+\tan x \sin u \partial_{u}, \quad Q_{3}=-\sin u \partial_{x}+\tan x \cos u \partial_{u}$

Invariant equation:

$$
u_{t}=\sqrt{\sec ^{2} x+u_{x}^{2}} \tilde{F}\left(t,\left(u_{x x} \cos x-\left(2+u_{x}^{2} \cos ^{2} x\right) u_{x} \sin x\right)\left(1+u_{x}^{2} \cos ^{2} x\right)^{-\frac{3}{2}}\right) .
$$

\section{Algebra $A_{3.8}$ :}

1. Realization

$$
Q_{1}=\partial_{u}, \quad Q_{2}=x \partial_{u}, \quad Q_{3}=-\left(x^{2}+1\right) \partial_{x}-x u \partial_{u} .
$$

Invariant equation

$$
u_{t}=\sqrt{1+x^{2}} \tilde{F}\left(t, u_{x x}\left(1+x^{2}\right)^{\frac{3}{2}}\right) .
$$

2. Realization

$Q_{1}=\partial_{u}, \quad Q_{2}=-\tan (t+x) \partial_{u}, \quad Q_{3}=\partial_{t}+\tan (t+x) u \partial_{u}$

Invariant equation

$$
u_{t}=u_{x}+\sec (t+x) F\left(x, u_{x x} \cos (t+x)-2 u_{x} \sin (t+x)\right) .
$$


Algebra $A_{3.9}$ :

1. Realization

$Q_{1}=\partial_{u}, \quad Q_{2}=x \partial_{u}, \quad Q_{3}=-\left(x^{2}+1\right) \partial_{x}+(q-x) u \partial_{u}, \quad q \neq 0$.

Invariant equation

$$
u_{t}=\mathrm{e}^{-q \arctan x} \sqrt{1+x^{2}} \tilde{F}\left(t, u_{x x} \mathrm{e}^{q \arctan x}\left(1+x^{2}\right)^{\frac{3}{2}}\right) .
$$

2. Realization

$$
Q_{1}=\partial_{u}, \quad Q_{2}=-\tan (t+x) \partial_{u}, \quad Q_{3}=\partial_{t}+(q+\tan (t+x)) u \partial_{u}, \quad q \neq 0 .
$$

Invariant equation

$$
u_{t}=u_{x}+\sec (t+x) \mathrm{e}^{q t} \tilde{F}\left(x, \mathrm{e}^{-q t}\left(u_{x x} \cos (t+x)-2 u_{x} \sin (t+x)\right)\right) .
$$

Differentiating any of the above equations by $x$ and replacing $u_{x}$ with $v$ yields an equation of the form (6) that admits a quasilocal symmetry. Consider, as an example, equation

$$
u_{t}=u_{x}+\sec (t+x) F\left(x, u_{x x} \cos (t+x)-2 u_{x} \sin (t+x)\right)
$$

which is invariant with respect to the algebra $\left\langle\partial_{t}+\tan (t+x) u \partial_{u}\right\rangle$. The corresponding one-parameter transformation group is

$$
\begin{aligned}
& t^{\prime}=t, \\
& x^{\prime}=x, \\
& u^{\prime}=u \sec (t+x+\theta),
\end{aligned}
$$

where $\theta \in \mathbb{R}$ is the group parameter. Computation of the first prolongation of the above formulas yields

$$
u_{x^{\prime}}^{\prime}=u_{x} \sec (t+x+\theta)+u \sec (t+x+\theta) \tan (t+x+\theta)
$$

or

$$
v_{x^{\prime}}^{\prime}\left(t^{\prime}, x^{\prime}\right)=(v(t, x)+u(t, x) \tan (t+x+\theta)) \sec (t+x+\theta),
$$

where $u(t, x)=\partial^{-1} v(t, x)$. The corresponding equation for $v=v(t, x)$ is

$$
v_{t}=v_{x}+\sec (t+x)\left(\tan (t+x) \tilde{F}+\tilde{F}_{\omega_{1}}\right)+\left(v_{x x}-3 \tan (t+x) v_{x}-2 v\right) \tilde{F}_{\omega_{2}} .
$$

Here $\tilde{F}$ is an arbitrary smooth function of the variables

$$
\omega_{1}=x \text { and } \omega_{2}=\cos (t+x) v_{x}-2 \sin (t+x) v .
$$

[1] Ovsiannikov L.V., Group analysis of differential equations, New York, Academic Press, 1982.

[2] Bluman G., Cole J., Similarity methods for differential equations, Berlin, Springer, 1974.

[3] Olver P., Applications of Lie groups to differential equations, New York, Springer, 1986.

[4] Fushchych W., Zhdanov R., Symmetries and exact solutions of nonlinear spinor equations, Kyiv, Mathematical Ukraina Publisher, 1997.

[5] Ovsiannikov L.V., Group properties of nonlinear heat equation, Dokl. AN SSSR, 1959, V.125, $492-495$ (in Russian). 
[6] Dorodnitsyn V.A., On invariant solutions of non-linear heat equation with a source, Zhurn. Vych. Matem. Matem. Fiziki, 1982, V.22, 1393-1400 (in Russian).

[7] Oron A., Rosenau Ph., Some symmetries of the nonlinear heat and wave equations, Phys. Lett. A, 1986, V.118, 172-176.

[8] Akhatov I.Sh., Gazizov R.K., Ibragimov N.H., Group classification of equations of nonlinear filtration, Dokl. AN SSSR, 1987, V.293, 1033-1035.

[9] Edwards M.P., Classical symmetry reductions of nonlinear diffusion-convection equations, Phys. Lett. A, 1994, V.190, 149-154.

[10] Yung C.M., Verburg K., Baveye P., Group classification and symmetry reductions of the non-linear diffusionconvection equation $u_{t}=\left(D(u) u_{x}\right)_{x}-K^{\prime}(u) u_{x}$, Int. J. Non-Linear Mech., 1994, V.29, 273-278.

[11] Pallikaros C., Sophocleous C., On point transformations of generalized nonlinear diffusion equations, J. Phys. A: Math. Gen., 1995, V.28, 6459-6465.

[12] Gandarias M.L., Classical point symmetries of a porous medium equation, J. Phys. A: Math. Gen., 1996, V.29, 607-633.

[13] Cherniha R., Serov M., Symmetries, ansätze and exact solutions of nonlinear second-order evolutions equations with convection terms, European J. Appl. Math., 1998, V.9, 527-542.

[14] El-labany S.K., Elhanbaly A.M., Sabry R., Group classification and symmetry reduction of variable coefficient nonlinear diffusion-convection equation, J. Phys A: Math. Gen., 2002, V.35, 8055-8063.

[15] Güngör F., Group classification and exact solutions of a radially symmetric porous-medium equation, Int. J. Non-Linear Mech., 2002, V.37, 245-255.

[16] Reid G.J., Finding abstract Lie symmetry algebras of differential equations without integrating determining equations, European J. Appl. Math., 1991, V.2, 319-340.

[17] Mubarakzjanov G.M., On solvable Lie algebras, Izv. Vyssh. Uchebn. Zaved. Matematika, 1963, N 1 (32), 114-123 (in Russian).

[18] Mubarakzjanov G.M., The classification of the real structure of five-dimensional Lie algebras, Izv. Vyssh. Uchebn. Zaved. Matematika, 1963, N 3 (34), 99-105 (in Russian).

[19] Fushchych W.I., Symmetry in mathematical physics problems, in Algebraic-Theoretical Studies in Mathematical Physics, Kyiv, Inst. of Math. Acad. of Sci. Ukraine, 1981, 6-28.

[20] Gagnon L., Winternitz P., Symmetry classes of variable coefficient nonlinear Schrödinger equations, J. Phys. A: Math. Gen., 1993, V.26, 7061-7076.

[21] Zhdanov R.Z., Roman O.V., On preliminary symmetry classification of nonlinear Schrödinger equation with some applications of Doebner-Goldin models, Rep. Math. Phys., 2000, V.45, 273-291.

[22] Zhdanov R.Z., Lahno V.I., Group classification of heat conductivity equations with a nonlinear source, J. Phys. A: Math. Gen., 1999, V.32, 7405-7418.

[23] Basarab-Horwath P., Lahno V., Zhdanov R., The structure of Lie algebras and the classification problem for partial differential equations, Acta Appl. Math., 2001, V.69, 43-94.

[24] Güngör F., Lagno V.I., Zhdanov R.Z., Symmetry classification of KdV-type nonlinear evolution equations, J. Math. Phys., 2004, V.45, 2280-2313.

[25] Lahno V., Zhdanov R., Group classification of nonlinear wave equations, J. Math. Phys., 2005, V.46, 053301, 37 pages.

[26] Lie S., On integration of a class of linear differential equations by means of definite integrals, Arch. Math., 1981, V.6, 328-368 (in German).

[27] Akhatov I.S., Gazizov R.K., Ibragimov N.K., Nonlocal symmetries: A heuristic approach, J. Soviet Math., 1991, V.55, 1401-1450.

[28] Meirmanov A.M., Pukhnachov V.M., Shmarev S.I., Evolution equations and Lagrangian coordinates, Berlin, Walter de Gruyter, 1997. 